\title{
Interdisciplinary management of a severely compromised periodontal patient - Case report
}

Coimbra J1, Sousa $A^{1}$, Pinhão Ferreira $A^{1}$, Coelho $V^{1}$, Pinhão Ferreira $A^{1}$

\section{Affiliations:}

1. Ortopóvoa, Ida

Póvoa de Varzim, Portugal

\section{Corresponding author:}

João Coimbra

coimbrajperio@gmail.com

\section{ABSTRACT}

Purpose: The aim of this case report is to suggest and discuss an up-to-date interdisciplinary approach to a complex periodontal case, adjusting different treatment strategies intra-individually based on the biological, functional and aesthetic dimensions of each patient.

Case report: The case refers to a 42 -year-old healthy male patient with IIIB generalized periodontitis. After cause-related therapy, the patient was submitted to periodontal resective surgery with fibre retention technique in the maxilla and surgical regenerative procedures in the posterior regions of the mandible with enamel matrix derivative and bovine xenograft. After Supportive Periodontal Therapy (SPT) institution the patient went through an aesthetic rehabilitation phase to minimize the disease sequelae. Conclusion: Modern evidence-based periodontal treatment options must be sitespecific oriented in order to achieve the best clinical outcomes. A contemporary vision of periodontal treatment must go beyond the moment of establishment of an individualized SPT programme and these restorative solutions are very powerful tools to give back confidence and increase the self-esteem of our patients.

\section{KEYWORDS}

Periodontitis, surgical regenerative procedure, full-thickness flap, aesthetic rehabilitation 


\section{INTRODUCTION}

Periodontitis is a chronic multifactorial disease initiated by bacterial microorganisms and characterized by severe chronic inflammation that leads to progressive destruction of the tooth supporting apparatus, tooth loss and eventually to masticatory dysfunction. ${ }^{1}$

The primary goal of the periodontal therapy is to arrest the inflammatory disease process and to manage the possible risk factors associated with it, allowing maintenance of a functional dentition for a lifetime. ${ }^{2}$

Besides saving teeth, patients with periodontitis are nowadays often seeking to address the sequelae that frequently arise from the disease progression, forcing the clinician to face demanding challenges, especially when the aesthetic zone is committed. ${ }^{3}$

\section{CASE REPORT}

This is the case of a 42-year-old male patient with no relevant medical conditions, complaining about gingival bleeding and aesthetically unpleasant smile (Figure 1, a-c).

A clinical examination was carried out and a panoramic radiography was taken (Figure 2). These evaluations suggested the further need for a full mouth meticulous assessment of the clinical attachment level through periodontal probing, as severe periodontal destruction was apparent.

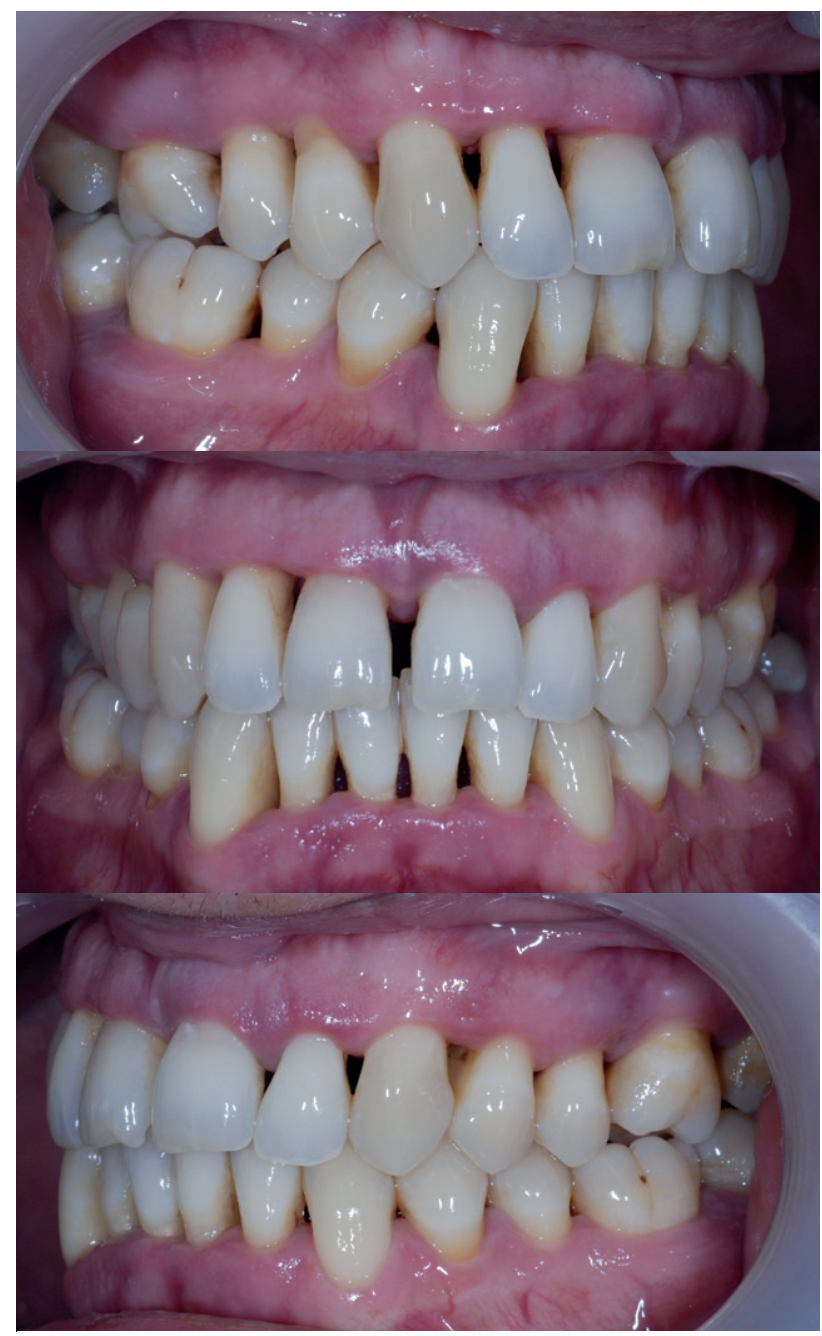

Figure 1 a-c. Initial intra-oral photographs at the moment of the first visit

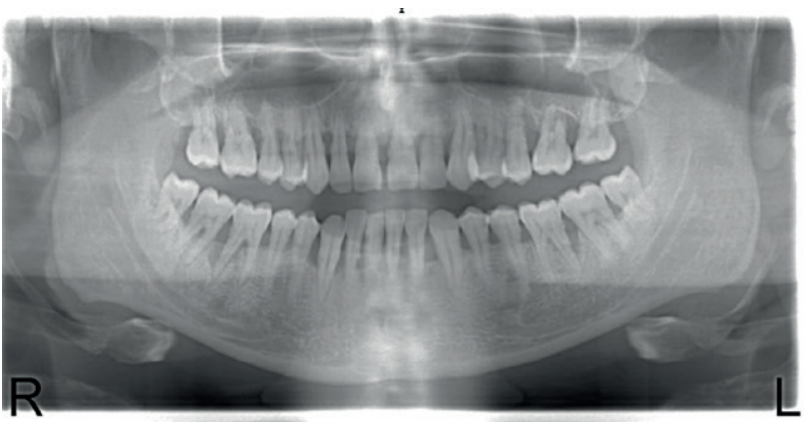

Figure 2. Initial panoramic radiograph 
An electronic probe was used (Florida Probe Corporation, USA) with a PD Probe tip with a diameter of $0,45 \mathrm{~mm}$. Records from the obtained periodontal chart (Table I) showed that $50 \%$ of the probed sites had at least $5 \mathrm{~mm}$ clinical attachment loss (CAL) and also that $19 \%$ presented probing depths $\geq 6 \mathrm{~mm}$. Likewise, bleeding on probing was present in $66 \%$ of the sites. Tooth mobility was found on teeth 25, 46, 47, 48 (grade I) and 42, 41, 31 (grade II). No teeth were lost due to periodontitis progression. According to the new classification scheme for periodontal and peri-implant diseases and conditions established by the 2017 World Workshop on the Classification of Periodontal and Periimplant Diseases and Conditions, this case matched a stage III, grade B, generalized periodontitis.,

Therapy began with a conventional cause related phase, providing the patient proper oral-hygiene instructions and motivation. Due to reported discomfort while chewing, splinting of lower incisors for better occlusal stabilization during therapy was initially accomplished. Afterwards, supra-gingival ultrasonic (Airflow Prophylaxis Master, EMS S.A., Switzerland) calculus debridement and delicate single session scaling and root planning (After five, Mini five, Hu-friedy Inc, USA) were performed to eliminate supra and sub gingival calculus and biofilm, thus leading to reduce signs of inflammation. Twice a day $0,12 \%$ chlorohexidine mouth rinse (Bexident ${ }^{\circ}$, ISDIN, Spain) was prescribed for a week as a coadjutant of the therapy. No antibiotics were prescribed. The use of an electronic toothbrush with timer and pressure sensor at home was prescribed to improve patient's compliance and plaque control skills.

Three months later the patient was re-evaluated, and four surgical interventions were planned for residual active pockets elimination. In the maxilla, periodontal resective surgeries with apically repositioned flaps were planned in the posterior teeth of the $1^{\text {st }}$ and $2^{\text {nd }}$ quadrants; in the mandible, specific regenerative approaches were proposed in the posterior teeth of the $3^{\text {rd }}$ and $4^{\text {th }}$ quadrants, not only to recover some lost periodontal attachment that originated angulated intrabony defects, but also to try to treat a grade II furcation lesion on tooth 46 .

The elected technique for the upper jaw interventions was the fibre retention osseous surgery, as proposed by Carnevale in 2007. ${ }^{6}$ This technique introduced some major modifications, when compared to the one proposed by Ochsenbein in 1958, allowing minimal ostectomy, preserving the supracrestal fibres

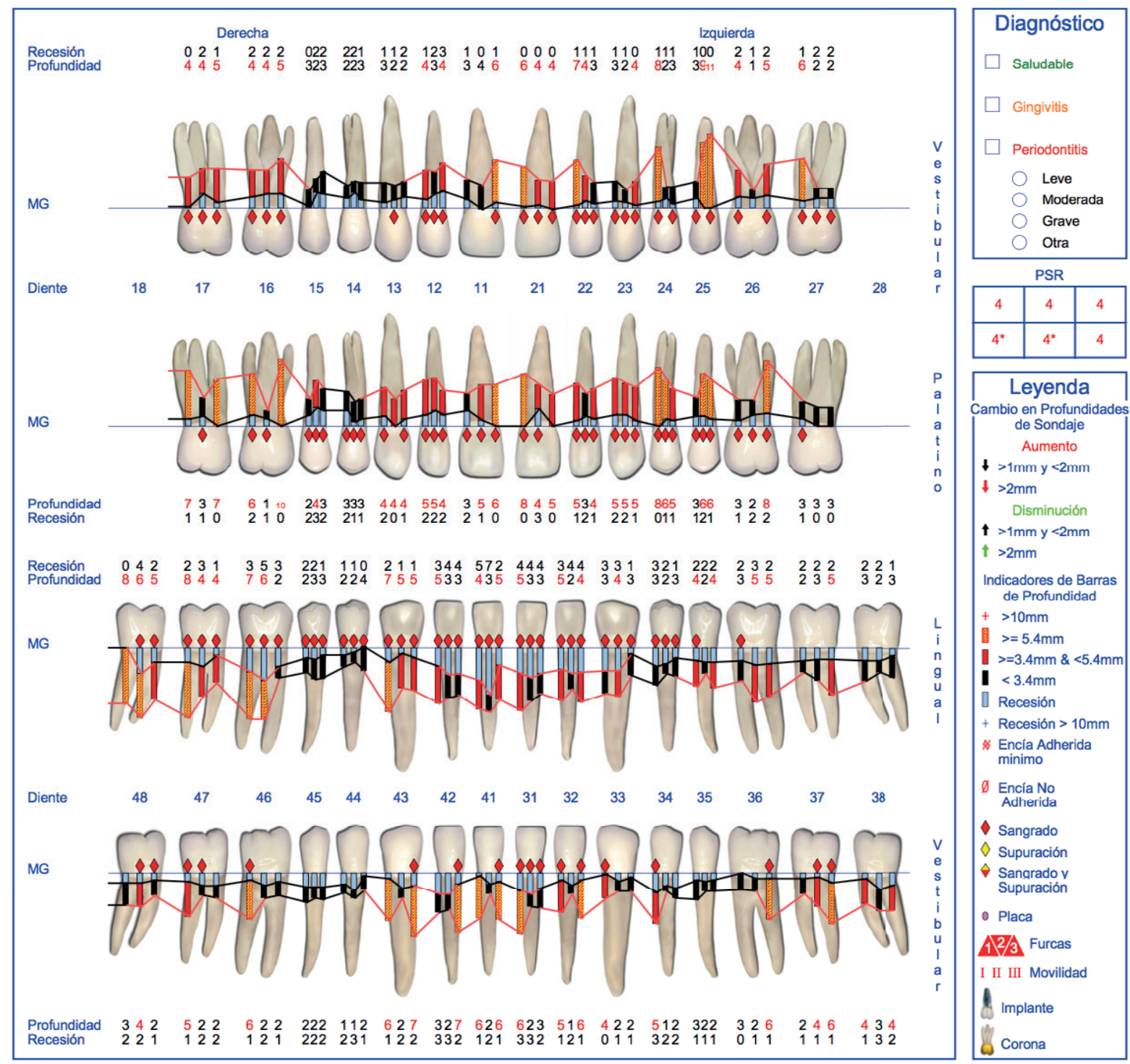

Table I. Initial periodontal chart (Florida Probe Coorporation, USA) 
invested in the root cementum and thus placing the deepest part of the intrabony defect in a more coronal position., $2,6,7$

A paramarginal incision guided by probing depths together with the apico-coronal height of the keratinized tissue was performed in both buccal and palatal sides (Figure 3, 4) with a 15C blade. A split-thickness flap, evenly dimensioned from distal to mesial, was prepared beyond the mucogingival junction in the buccal

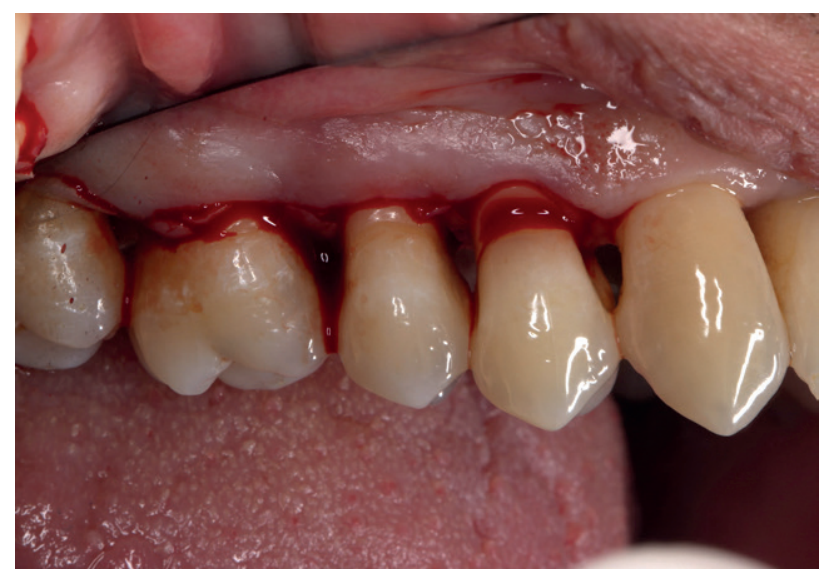

Figure 3. Primary paramarginal incision in the buccal side

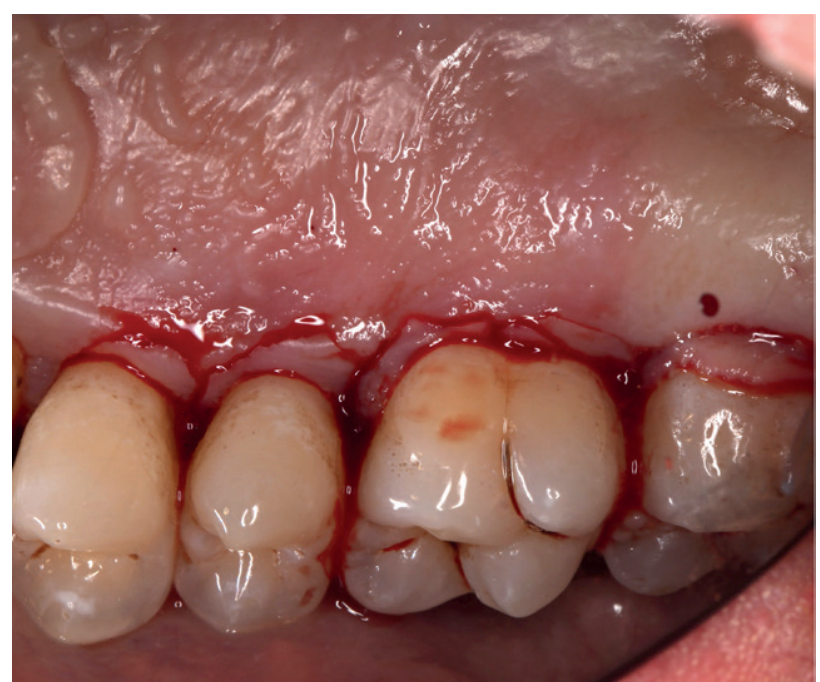

Figure 4. Primary paramarginal incision in the palatal side. Notice the overcut of the incision in the papillae region

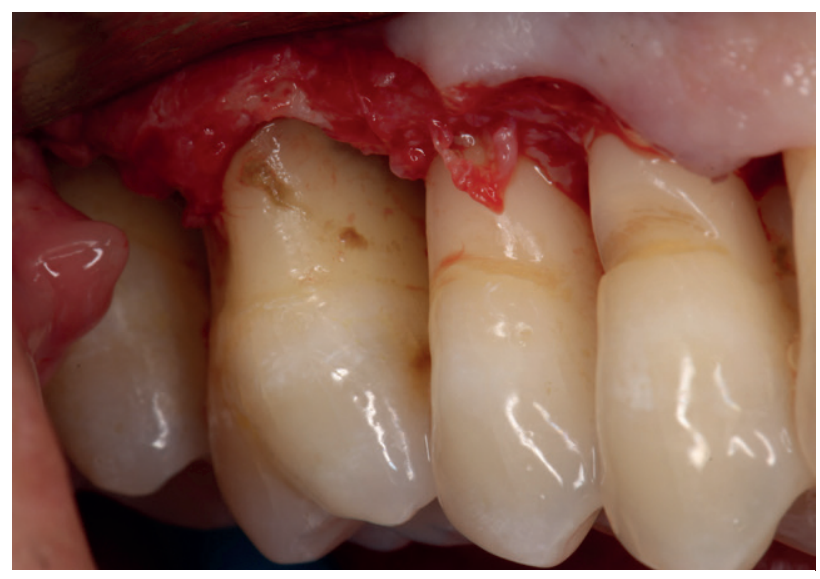

Figure 5. Split-thickness flap was prepared in the buccal side and subgingival calculus could be identified side (Figure 5). Splitting the flap allowed greater mobility so that it could be apically positioned afterwards.

All the marginal soft tissue coronal to the primary incision was removed with a similar blade. At the palatal area, the split-thickness flap was extended 6 to $8 \mathrm{~mm}$ apical to the cementoenamel junction (CEJ), resulting in a thinned palatal flap. At that point, an incision made perpendicular to the bone enabled the deepest portion of the connective tissue with the periosteum to be removed and made it possible for the flap to rotate apically (Figure 6).

In the interproximal areas, soft tissue was removed with a 12D blade. The identification of the fibre system was done through the use of magnifying loupes and, when needed, a periodontal probe, to gently discriminate attached from granulation tissue. Non-attached soft tissue was carefully removed with the blade; fibres were left connected to the root cementum. ${ }^{2,6}$ Calculus was identified and cautiously removed with ultrasonic instruments (Airflow ${ }^{\circ}$ Prophylaxis Master, EMS S.A., Switzerland), minicurettes (Hu-friedy Inc, USA) and erythritol powder (Airflow ${ }^{\circ}$ Plus, EMS S.A., Switzerland) was used to help completing biofilm removal (Figure 7).

Finally, both buccal and palatal flaps were sutured apically with nylon 6-0 sutures (Dafilon', B-braun surgical S.A., Spain)

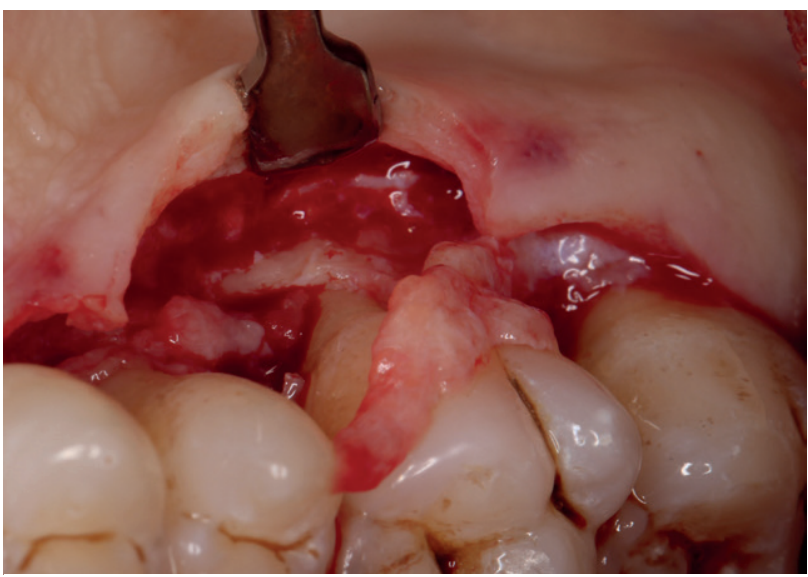

Figure 6. Soft tissue removal from the palate, after split-thickness flap preparation. This tissue is to be eliminated through a full thickness elevation

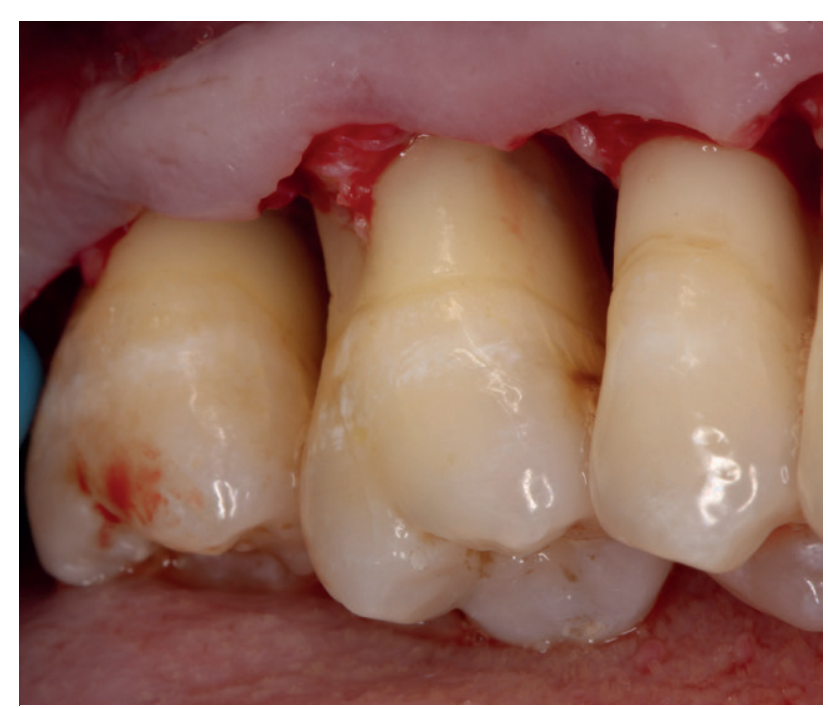

Figure 7. Clinical presentation after root debridement in the buccal side. Notice the attached fibres in the furcation area, which were preserved to avoid loss of attachment, bone resorption and development of lesion 
anchored to the periosteum in both sides, allowing good scalloped adaptation and secondary intention healing in the interproximal spaces (Figure 8-9).

In the lower jaw, the treatment strategy was different. Periodontal regeneration procedures were indicated in the molar areas of the $3^{\text {rd }}$ and $4^{\text {th }}$ quadrants due to the presence of well-defined isolated deep intrabony defects, both on buccal and lingual aspects of the interdental spaces of teeth $46 / 47$ (6-7 mm probing pocket depths with lingual furcation Class II lesion, according to Hamp in $1975,{ }^{8}$ and CAL $10-11 \mathrm{~mm}$ on tooth 46) and 36/37 (probing pocket depths $5-6 \mathrm{~mm}$ and CAL $7-8 \mathrm{~mm}$ on tooth 36 ).

The Minimally Invasive Surgical Technique (MIST), as proposed by Cortellini in 2007 was the selected approach for each of these defects. This technique has been specifically designed to treat intrabony defects with periodontal regeneration. ${ }^{9}$

In the $4^{\text {th }}$ quadrant (Figure 10), the surgical procedure began with a Modified Papilla Preservation Technique (MPPT), since the interdental papilla space was wider than $2 \mathrm{~mm} .{ }^{10}$ This incision was performed with a micro-blade (Micro Blade SR, Keydent, American Dental Systems GmbH, Germany). The inter-dental incision extended to buccal and lingual aspects of the two teeth adjacent to the defect. These incisions were strictly intra-sulcular to preserve all the height and width of the gingiva, and their mesio-distal extension was kept to a minimum to allow an apicocoronal gentle elevation of a full-thickness flap with a papilla elevator (H.Zepf Medical Instruments ${ }^{\circ} \mathrm{GmbH}$, Germany) that exposed sub-gingival calculus and biofilm. (Figure 11). ${ }^{9}$ Careful defect debridement was then accomplished with an ultrasonic scaler (Airflow Prophylaxis Master, EMS S.A., Switzerland), mini curettes (Hu-friedy Inc, USA) to specifically remove calculus and granulation tissue, and erythritol prophylaxis (Airflow ${ }^{\circ}$

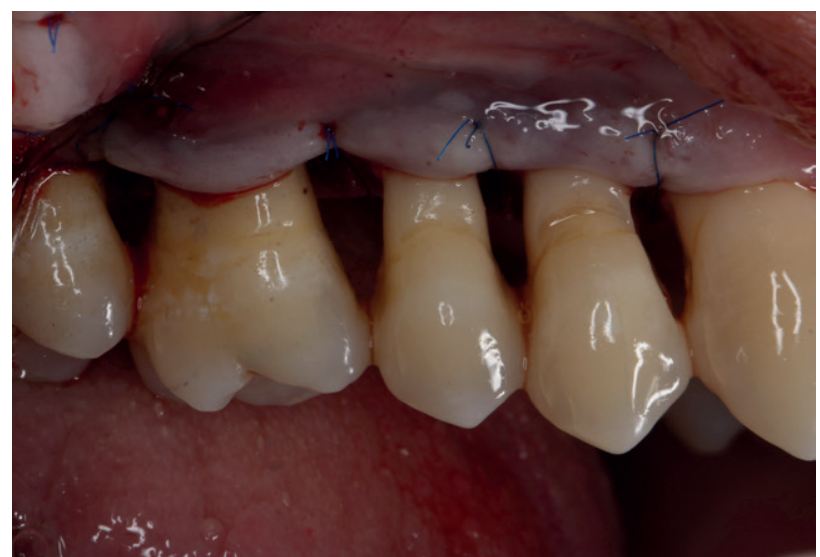

Figure 8. Final situation after apically positioning the flap, anchored in the periosteum with 6-0 nylon sutures

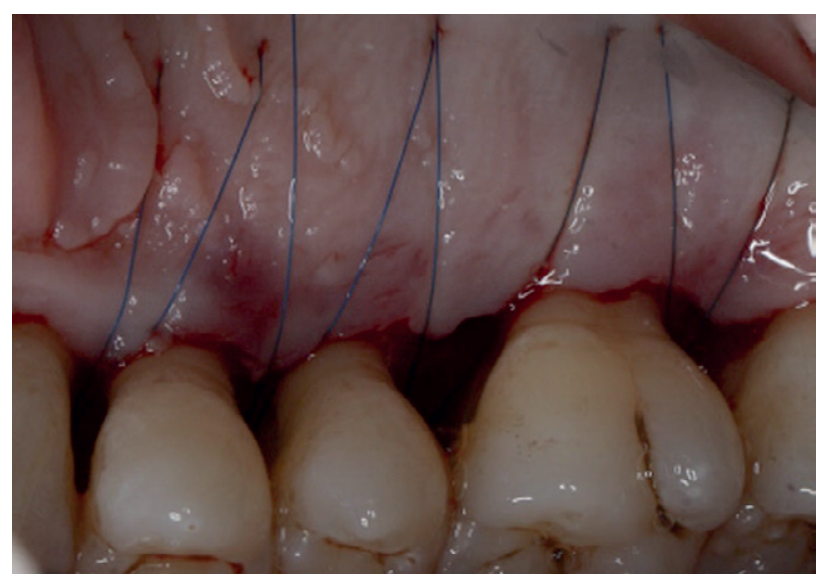

Figure 9. Monofilament 6-0 sutures anchoring and stabilizing the flap in the periosteum in the palatal side. The thinning of the flap allowed its apical rotation and crown lengthening sub and supragingival tip, Airflow Plus, EMS S.A., Switzerland). Supra-crestal attachment fibres in unaffected lingual and buccal crests were left untouched, connected to the root cementum, in order to prevent additional loss of attachment and improve flap stability (Figure 12). ${ }^{6}$

In the lingual aspect, flap elevation had to go deeper until the lingual bone crest was reached. Class II furcation lesion was identified on tooth 46 and soft tissue recession was also present in the gingival margin. Hence, prior to periodontal regeneration, soft tissue improvement in this side was also indicated, thickening the gingival margin, enhancing wound stability and therefore protecting the regenerated area during the healing process. ${ }^{11,12}$

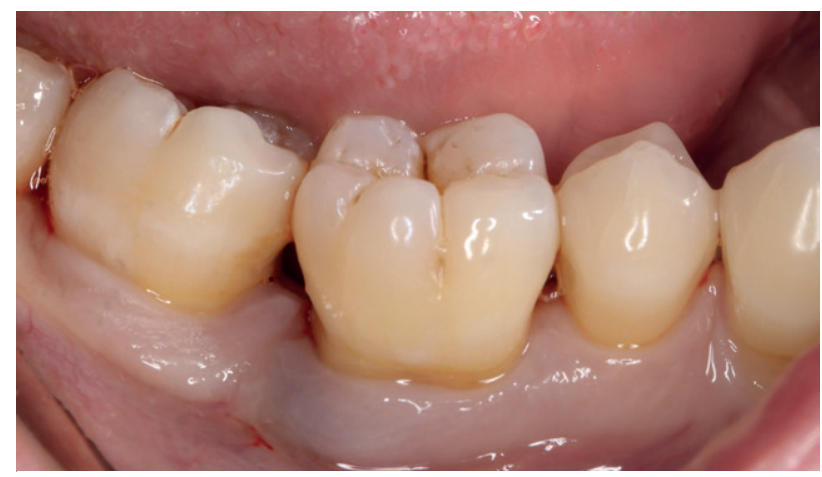

Figure 10. Pre-surgical situation in the buccal aspect of the $4^{\text {th }}$ quadrant

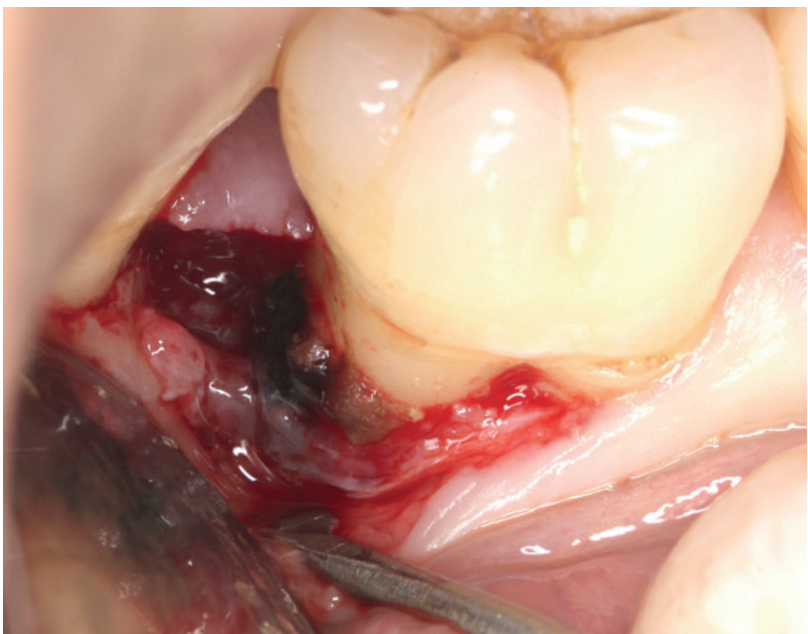

Figure 11. Full-thickness flap elevated in the buccal side with a papilla elevator, after intra-sulcular incision. Calculus and granulation tissue are exposed. Apically, flap elevation stops at 1-2mm exposed bone

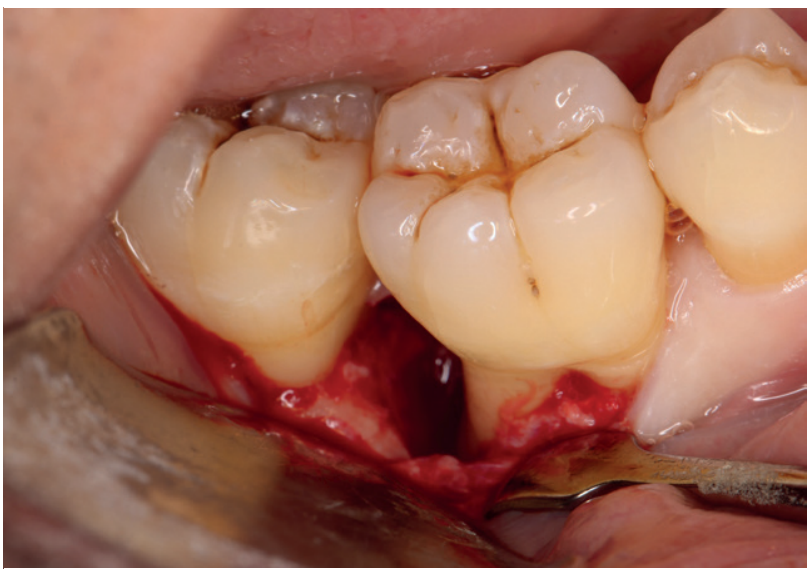

Figure 12. After root debridement, the intrabony component of the defect is exposed. Connective tissue attachment in the buccal bone crest remains connected to the cementum to enhance wound primary closure without further bone resorption. Identification of these fibres is performed with a periodontal probe and magnification loupes. non-attached soft tissue represents granulation tissue and therefore needs to be removed 
A connective tissue graft from the tuberosity was harvested and stabilized with one single interrupted suture in the internal wall of the lingual flap (Figure 13). After proper debridement of the affected lingual surfaces, periodontal regeneration was carried out with the use of 24\% EDTA gel (PrefGel', Straumann Institute AG, Switzerland), enamel matrix derivate (Emdogain", Straumann Institute AG, Switzerland) and finally particulate bone xenograft (Cerabone, Botiss Dental GmbH, Germany)..$^{13,14}$ The flap was then closed with one double-sling 6-0 nylon suture (Dafilon", B-braun Surgical S.A., Spain) in the centre of the interdental papilla, providing perfect passive adaptation of the soft tissue (Figure14). Similar procedure was executed in the $3^{\text {rd }}$ quadrant. After surgical therapy was carried out, the 3-month followup situation is shown in Figure 15. At this point, supportive periodontal therapy (SPT) was planned, according to the patient's grade B risk profile, with regular visits every three months for the first two years and restorative and aesthetic treatment was initiated. ${ }^{4,15}$

After discussing with the patient about aesthetic sequelae and concerns, and having performed a facial analysis, the main issues were addressed with 4 bonded layered lithiumdisilicate veneers (IPS e.max Press, Ivoclar Vivadent, Schaan, Liechtenstein) on the four upper incisors and direct bonding of composite resin (Filtek ${ }^{\text {TM }}$ Supreme XTE, 3M, St. Paul, MN, USA) on the lower incisors and canines.

In this particular case, the patient's chief complaints were the black triangles on the upper and lower anterior teeth and a diastema on the upper central incisors. The facial analysis revealed, also, a reverse incisal curve in relation to the concavity of the lower lip in rest and smile position. ${ }^{16}$

To close the upper central diastema, it is advised that the emergence profile of the restoration is one that allows a good gingival support as well as an hygienic profile, avoiding food debris and biofilm accumulation. As suggested by Magne and Belser ${ }^{17}$, it was decided to place the restoration margins subgingivally in the interproximal aspects of the diastema; combined with the intention to change the incisal border placement in order to harmonize the incisal curve, the option for a ceramic material was the most indicated ${ }^{18,19}$, in this case, four bonded layered lithium-disilicate (IPS e.max ${ }^{\circ}$ Press, Ivoclar Vivadent, Schaan, Liechtenstein). A previous mock-up was prepared and discussed with the patient. The tooth preparation was made through the mock-up in order to minimize the removal of sound tooth structure..$^{20} \mathrm{As}$ is frequent in periodontal cases, there is a lengthened clinical crown with root (and cementum) exposure. In order to enhance the adhesion to this substrate, the hybridization of the root surfaces was performed after tooth preparation and a self-etch, two-step bonding system was used throughout this case (Clearfi ${ }^{\mathrm{TM}}$ SE Bond 2, Kuraray, Tokyo,

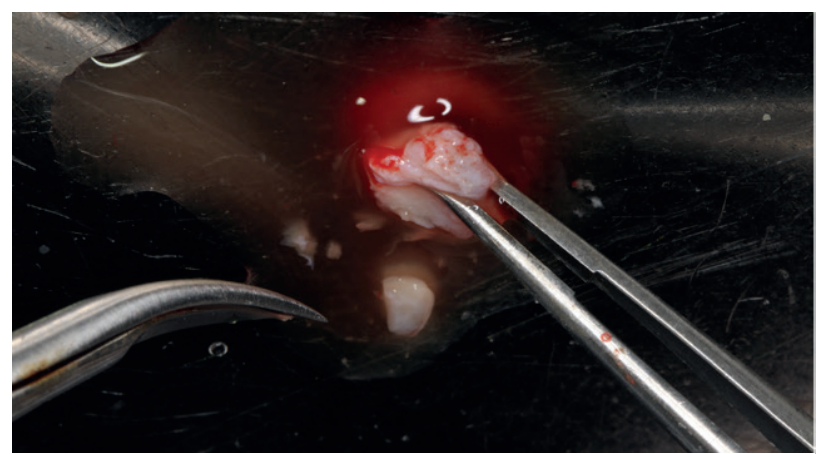

Figure 13. Connective tissue graft harvested from the tuberosity to improve soft tissue quality in the lingual marginal recession on tooth 46 . The thickening of the margin provided better wound stabilization and protected the regenerated furcation area
Japan) ${ }^{21,22}$ (Figure16). A light-cured resin cement was used to bond the ceramic veneers (Figure 17).

The restorative approach on the mandibular anterior teeth black triangles was for a direct bonding of composite resin one, as the

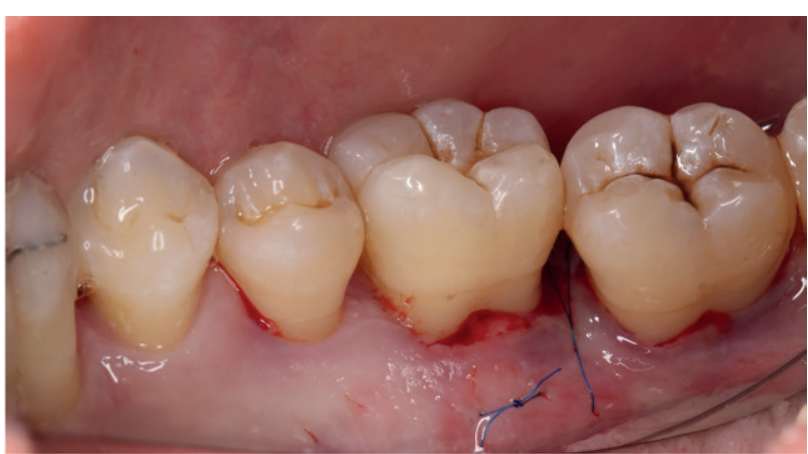

Figure 14. Lingual aspect of the $4^{\text {th }}$ quadrant after the flap was sutured with one double-sling 6-0 nylon suture. Perfect adaptation of the lingual and buccal flaps can be observed. The connective tissue graft is stabilized with a single interrupted suture and is perfectly adapted to the furcation entrance, improving soft tissue quality in that specific point and protecting the regeneration underneath during the healing phase

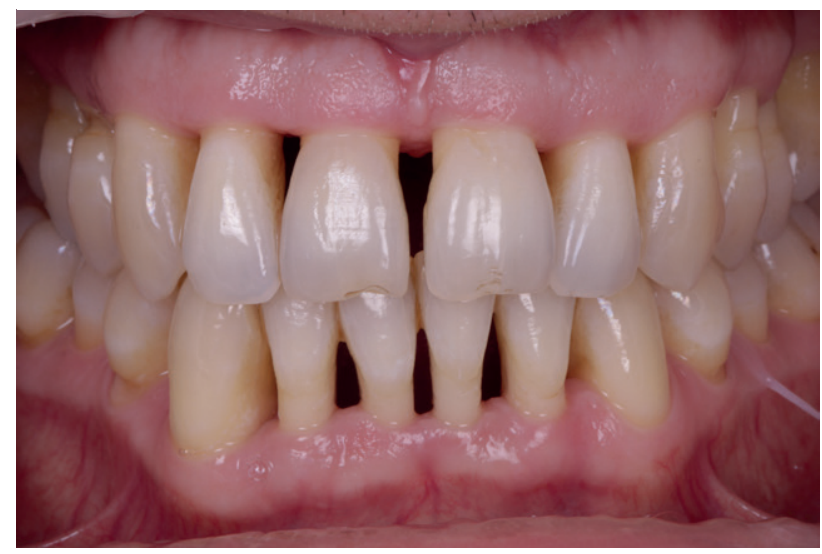

Figure 15. SPT at 3 months with no signs of active periodontal disease. Patient proves to be motivated and able to proceed to the aesthetic rehabilitation phase

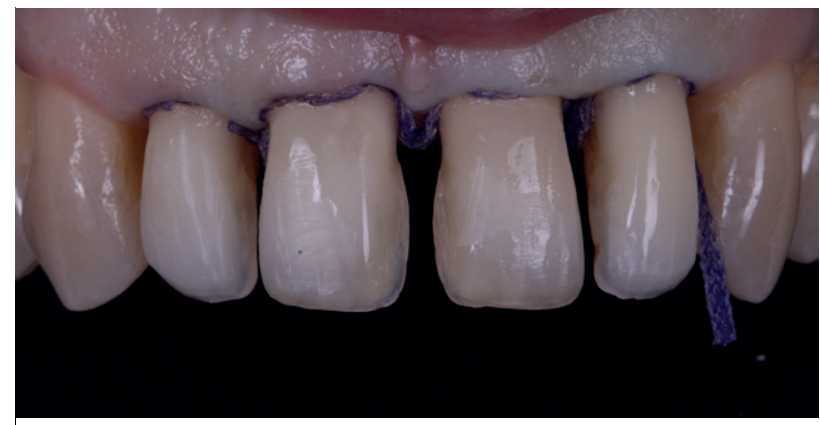

Figure 16. Maxillary incisors after tooth preparation and hybridization of the exposed root surface, immediately before the taking of the final impression

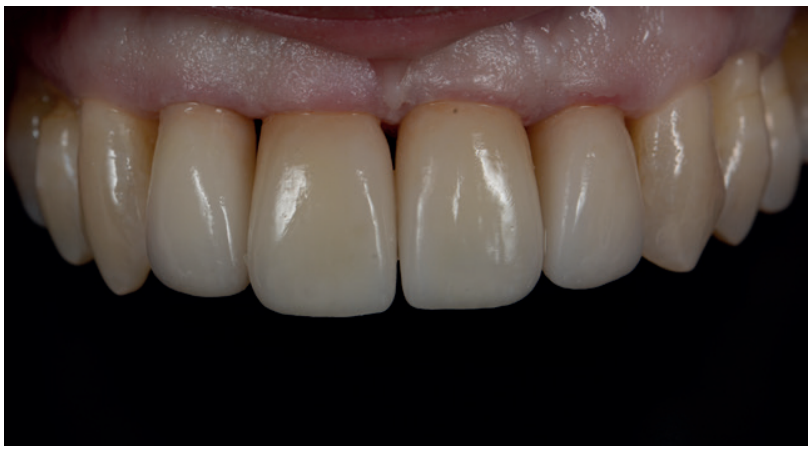

Figure 17. Final aspect on the delivery appointment, immediately after the removal of the rubber dam 
incisal borders were not to be changed and the aesthetic factor was less impactful, allowing for a supragingival restoration. ${ }^{3}$ The splinting wire was first removed in order to place a rubber dam (Nic Tone ${ }^{\circ}, \mathrm{MDC}$, Mexico). The teeth were thoroughly cleaned with sandblasting and mechanical action of mini curettes (Figure 18). Selective acid etching was performed on enamel with $37 \%$ phosphoric acid, and a self-etch, two-step adhesive was used on all surfaces involved. Composite resin was placed freehand, reshaping the teeth, closing the black triangles, keeping in mind the preservation of a freeway for the use of an interdental brush by the patient (Figure 20). The teeth were, then, again, splinted. At the 9-month follow up appointment, SPT was performed and control radiographs (Figures 20-21) and photos (Figures 22-23) were made. Figure 20 shows the intrabony defect and furcation lesion healing on tooth 46 , corroborating previously known data (23), while Figure 21 shows the corresponding scenario in the $3^{\text {rd }}$ quadrant.

Table II shows the 12-month periodontal chart at the 1-year recall visit.

\section{DISCUSSION}

Periodontitis is characterized by microbially-associated; host mediated inflammation that results in loss of periodontal attachment. This is detected as CAL by circumferential assessment of the erupted dentition with a standardized periodontal probe with reference to the CEJ. 5

The 2017 classification of the periodontal diseases ${ }^{4,5}$ provides a new framework that enables clinicians to address periodontal diseases not only according to its severity and complexity of management at presentation - staging - but also in relation to biological features of the disease, including history based analysis of the rate of disease progression, assessment of risk of further progression, anticipated poor outcomes of the treatment and assessment of the risk that the disease or its treatment may negatively affect the general health of the patient - grading. ${ }^{5}$ Staging comprises categories I to IV, whereas grading runs from A to $\mathrm{C}$ as the rate of progression gets higher.

In this case scenario, at stage III, periodontitis had produced

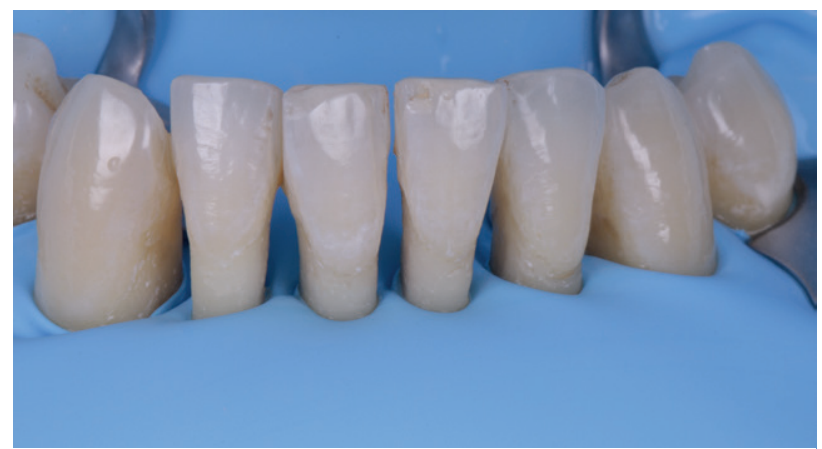

Figure 18. Mandibular anterior teeth with placed rubber dam after root surface cleaning

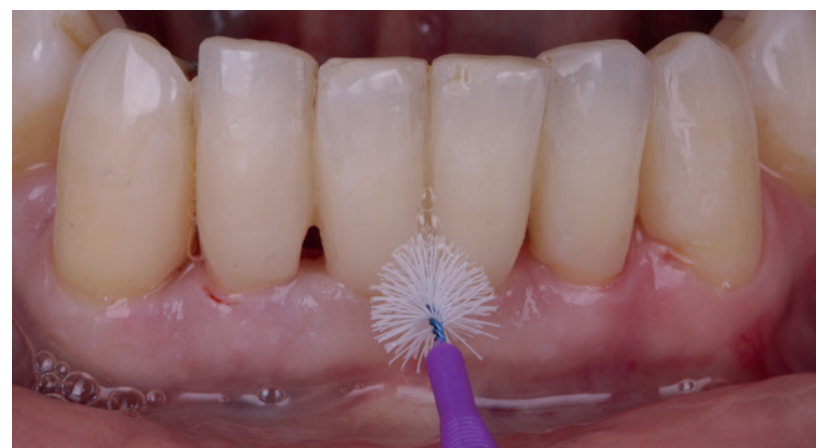

Figure 19. The finished interproximal restorations, with the reshaped black triangles. A freeway for the interdental brush was perfectly maintained

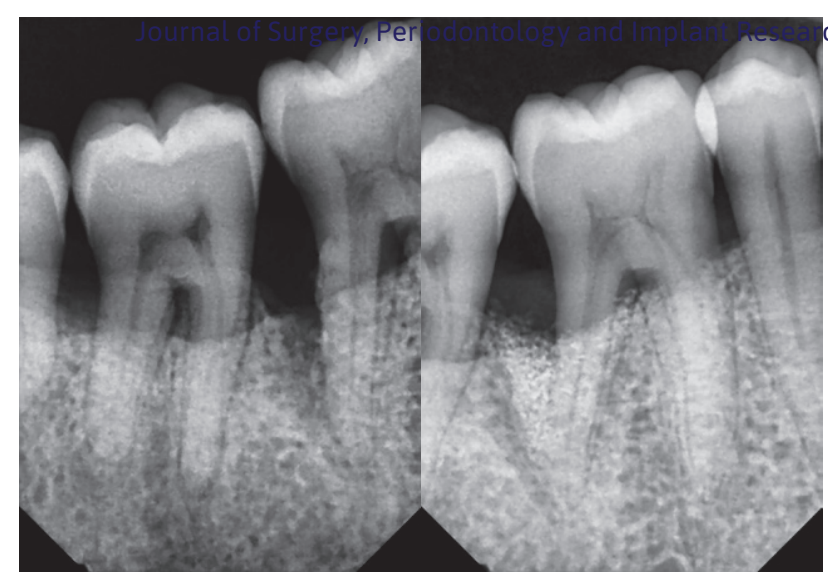

Figure 20. Radiographs of teeth $46 / 47$ at baseline and at 9 months follow up, after periodontal regenerative surgery

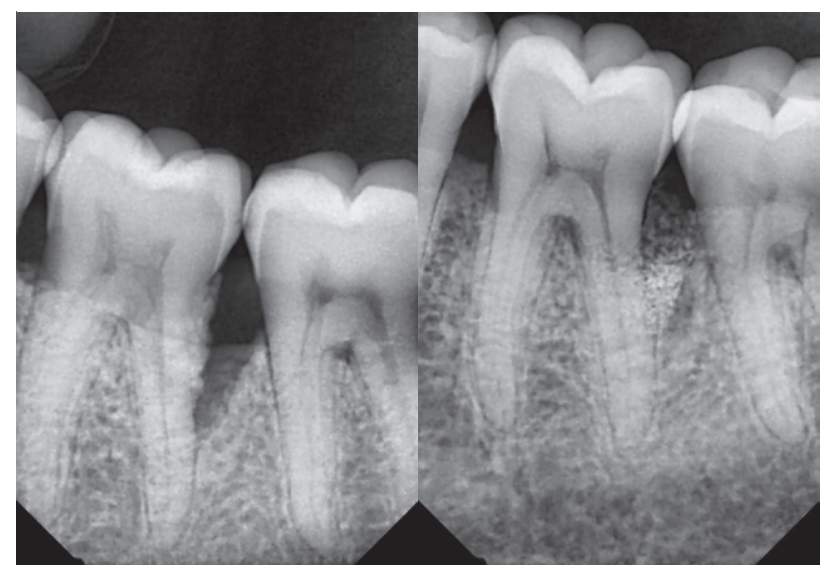

Figure 21. Radiographs of teeth $36 / 37$ at baseline and at 9 months follow up, after periodontal regenerative surgery. Notice the calculus presence on the distal aspect of the crown and root of tooth 36 , prior to surgical intervention

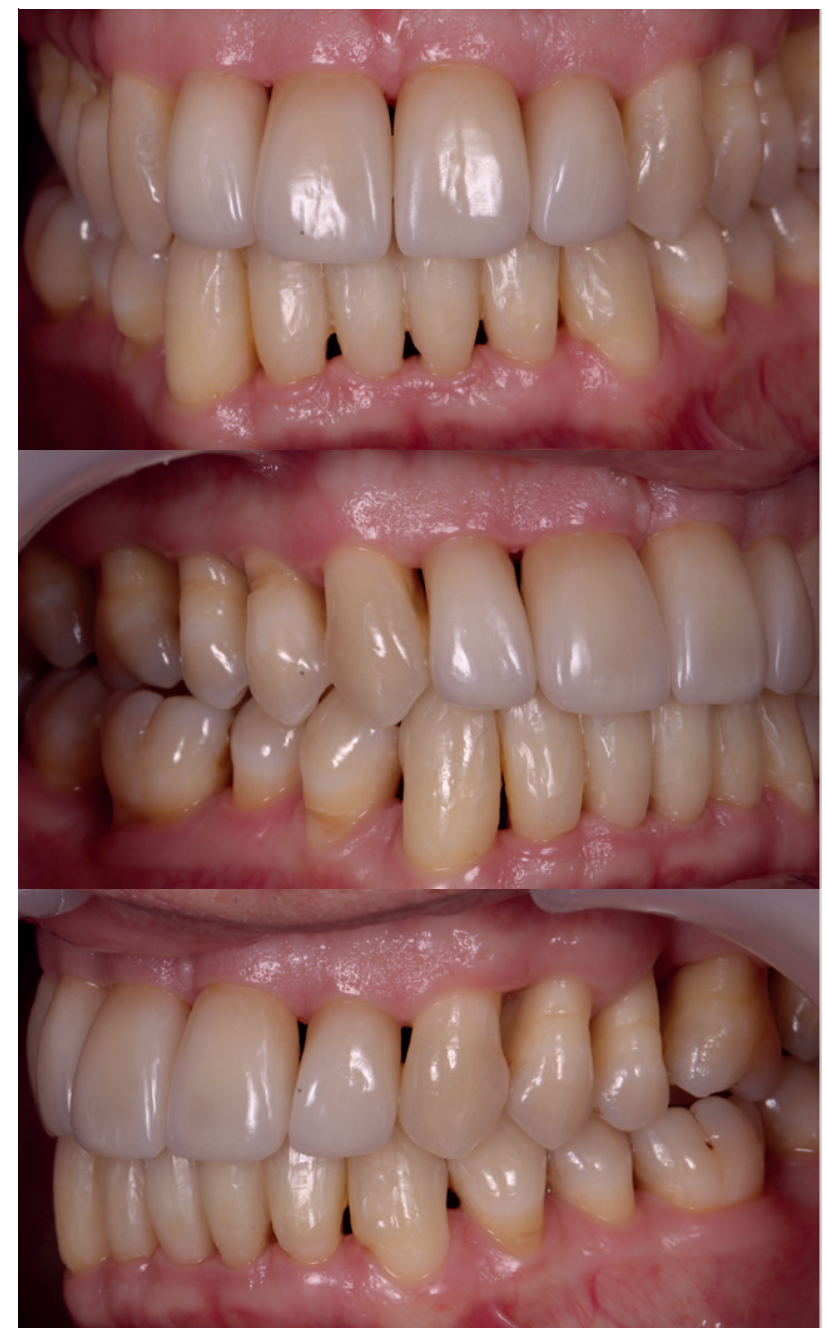

Figure 22 a-c. 9-month follow-up clinical stable situation with no signs of active periodontal disease and very low full mouth plaque score 


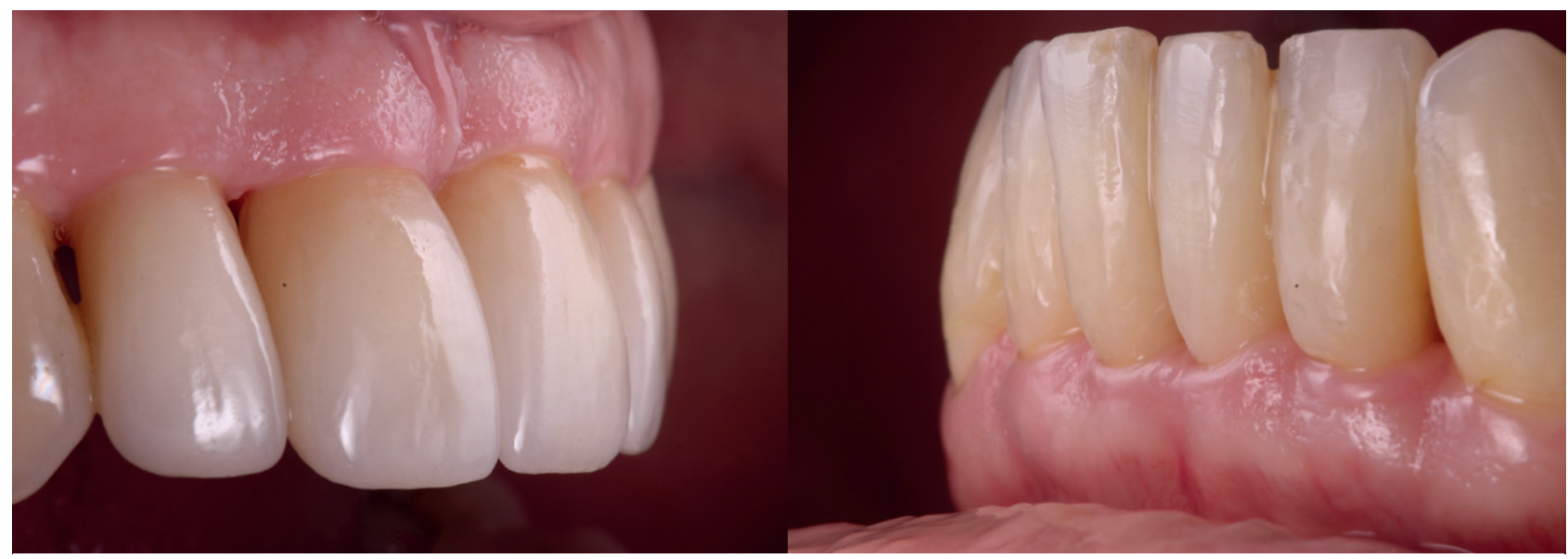

Figure 23 a-b. Detailed view of the maxillary and mandibular anterior region at 9-month follow-up, where a good biological integration of the performed restorative solutions can be shown, with no sign of inflammation and excellent plaque control skills shown by this motivated patient. Gingival health is achieved, even in a reduced periodontium

Fecha: July 02, 2019, 09:35

Visita Comparada con:

October 30, 2017, 14:04

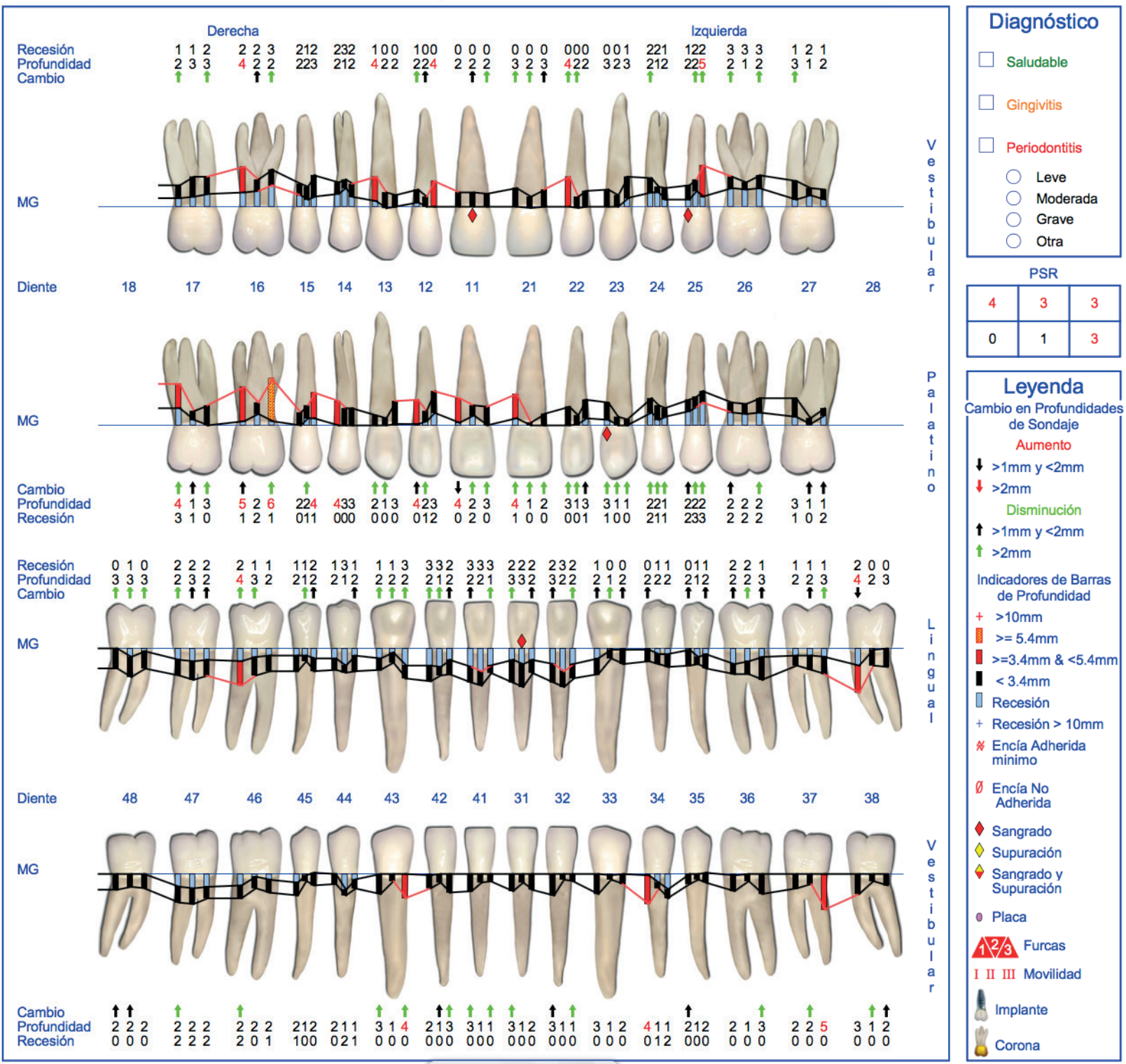

Table II. 12-month periodontal chart (Florida Probe Coorporation, USA) 
significant damage to the attachment apparatus, and, in the absence of advanced treatment, tooth loss may occur. Stage III is characterized by the presence of deep periodontal lesions that extend to the middle portion or apical third of the root and whose management is complicated by the presence of deep intrabony defects and class II or III furcation involvement. Nevertheless, despite the possibility of tooth loss, masticatory function is still preserved in these situations. ${ }^{5}$

Grading of periodontitis allows rate of progression to be considered. It can be modified by the presence of risk factors. ${ }^{5}$ In this case, although the percentage of radiographic bone loss as a function of age at the most affected tooth could suggest a rapid rate of progression, the presented destruction was commensurate with biofilm deposits. Being a non-smoker, non-diabetic patient who was motivated to follow the given instructions in order to enhance plaque control and reduce the inflammatory burden of the disease, the case was graded B.

The upper jaw was treated with periodontal resective surgery. Open flap debridement or apically positioned flaps with or without bone recontouring are generally performed for the treatment of moderate to advanced periodontal lesions, with $\leq 3 \mathrm{~mm}$ intrabony components, aiming to reduce probing pocket depths. In this case, periodontal resective technique was applied in conjunction with fibre retention, shifting the bottom of the defect to a more coronal position at the level of the connective tissue attachment. This approach considers mineralized and non-mineralized tissue as a unit and changes the classical vision of the osseous defect, resulting in more conservative removal of supportive bone., ${ }^{2,6}$ With this technique, since 2007 Carnevale has shown minimal pocket depths at the end of therapy, residual full mouth plaque and bleeding scores and low disease recurrence throughout a proper maintenance phase. ${ }^{2}$

In the lower jaw, regenerative procedures were carried out to treat deep intrabony isolated periodontal defects $(>3 \mathrm{~mm})$, where gaining attachment was a therapy end-point. The MIST is intended to reduce surgical trauma, increase flap and wound stability, allow stable, primary closure of the wound, reduce surgical chair time and minimize patient discomfort and side effects (9). This approach requires the use of microsurgical instruments, magnification devices and optimal illumination, making it possible to perform surgery with reduced flap reflection, thus empowering the healing process. ${ }^{9,24,25}$

To accomplish regeneration, enamel matrix derivate was mixed with a particulate bovine bone graft.13,14 The enamel matrix derivate has proven to be reliable in improving clinical parameters in the treatment of intrabony defects and furcation lesions alone or combined with a grafting material, providing more horizontal depth reduction of these lesions compared to barrier membranes. ${ }^{26,27}$

The most frequent aesthetic sequelae cause of concern to a periodontal patient are related to gingival recession and the collapse of the alveolar crest and interproximal tissue volume, which lead, frequently, to both lengthening of the clinical crown and the opening of black triangles. ${ }^{3}$

In this case, through stringent SPT performance, future tooth loss risk is minimal, as reduction of pocket depth following active therapy is expected to be stable over time with no persistent signs of inflammation. ${ }^{28}$

\section{CONCLUSION}

This clinical case has been reported to emphasize a comprehensive view of the periodontal disease management. Modern evidence-based treatment options must be site-specific oriented in order to achieve the best clinical outcomes.

Success relies not only in delivering accurate diagnosis, performing meticulous surgical procedures, using sensitive microsurgical and magnifying instruments, but also in succeeding to match patients' primary needs with further emotional ambitions.

A contemporary vision of periodontal treatment must go beyond the moment of establishment of an individualized supportive periodontal therapy programme. The above proposed restorative solutions are very powerful tools to be taken into account when overcoming treatment's sequelae from an aesthetical, minimally invasive, biomimetic standpoint, and this is of most importance to our patients.

\section{CONFLICT OF INTEREST}

The authors declares that there is no conflict of interest regarding the publication of this article. 


\section{REFERENCES}

1. Periodontology EFo. EFP Manifesto Perio and General Health. 2018

2. Carnevale G, Cairo F, Tonetti MS. Long-term effects of supportive therapy in periodontal patients treated with fibre retention osseous resective surgery. I: recurrence of pockets, bleeding on probing and tooth loss. J Clin Periodontol. 2007;34(4):334-41.

3. Ricci A, Ferraris F. A minimally invasive approach to restore function and esthetics in periodontally involved teeth. Eur J Esthet Dent. 2011;6(1):34-49.

4. Caton JG, Armitage G, Berglundh T, Chapple ILC, Jepsen S, Kornman KS, et al. A new classification scheme for periodontal and peri-implant diseases and conditions - Introduction and key changes from the 1999 classification. J Periodontol. 2018;89 Suppl 1:S1-S8

5. Tonetti MS, Greenwell H, Kornman KS. Staging and grading of periodontitis: Framework and proposal of a new classification and case definition. J Clin Periodontol. 2018;45 Suppl 20:S149-S61.

6. Carnevale G. Fibre retention osseous resective surgery: a novel conservative approach for pocket elimination. J Clin Periodontol. 2007;34(2):182-7.

7. Ochsenbein C. Osseous resection in periodontal susrgery. J Periodontol. 1958;29:15-26.

8. Hamp SE, Nyman S, Lindhe J. Periodontal treatment of multirooted teeth. Results after 5 years. J Clin Periodontol. 1975;2(3):126-35.

9. Cortellini P, Tonetti MS. A minimally invasive surgical technique with an enamel matrix derivative in the regenerative treatment of intra-bony defects: a novel approach to limit morbidity. J Clin Periodontol. 2007;34(1):87-93.

10. Cortellini P, Prato GP, Tonetti MS. The modified papilla preservation technique. A new surgical approach for interproximal regenerative procedures. J Periodontol. 1995;66(4):261-6.

11. Santoro G, Zucchelli G, Gherlone E. Combined Regenerative and Mucogingival Treatment of Deep Intrabony Defects Associated with Buccal Gingival Recession: Two Case Reports. Int J Periodontics Restorative Dent. 2016;36(6):849-57.

12. Zucchelli G, Bentivogli V, Ganz S, Bellone P, Mazzotti C. The connective tissue graft wall technique to improve root coverage and clinical attachment levels in lingual gingival defects. Int J Esthet Dent. 2016;11(4):538-48.

13. Artzi Z, Tal H, Platner O, Wasersprung N, Weinberg E, Slutzkey S, et al. Deproteinized bovine bone in association with guided tissue regeneration or enamel matrix derivatives procedures in aggressive periodontitis patients: a 1-year retrospective study. J Clin Periodontol. 2015;42(6):547-56.

14. Esposito M, Grusovin MG, Papanikolaou N, Coulthard P, Worthington HV. Enamel matrix derivative (Emdogain) for periodontal tissue regeneration in intrabony defects. A Cochrane systematic review. Eur J Oral Implantol. 2009;2(4):247-66.

15. Lang NP, Tonetti MS. Periodontal risk assessment (PRA) for patients in supportive periodontal therapy (SPT). Oral Health Prev Dent. 2003;1(1):7-16.

16. Fradeani M. Esthetic Rehabilitation in Fixed Prosthodontics: Quintessence Publishing Co. Ltd; 2004

17. Magne PB, U. Bonded Porcelain Restorations in the Anterior Dentition: A Biomimetic Approach: Quintessence Publishing Co. Ltd; 2002.

18. Peumans M, Van Meerbeek B, Lambrechts P, Vanherle G, Quirynen M. The influence of direct composite additions for the correction of tooth form and/or position on periodontal health. A retrospective study. J Periodontol. 1998;69(4):422-7.

19. Peumans M, Van Meerbeek B, Lambrechts P, Vanherle G. Porcelain veneers: a review of the literature. J Dent. 2000;28(3):163-77.

20. Gurel G. Porcelain laminate veneers: minimal tooth preparation by design. Dent Clin North Am. 2007;51(2):419-31, ix.

21. De Munck J, Van Landuyt K, Peumans M, Poitevin A, Lambrechts P, Braem M, et al. A critical review of the durability of adhesion to tooth tissue: methods and results. J Dent Res. 2005;84(2):118-32.

22. Tanaka S, Sugaya T, Kawanami M, Nodasaka Y, Yamamoto T, Noguchi H, et al. Hybrid layer seals the cementum/4-META/MMA-TBB resin interface. J Biomed Mater Res B Appl Biomater. 2007;80(1):140-5

23. Reddy MS, Aichelmann-Reidy ME, Avila-Ortiz G, Klokkevold PR, Murphy KG, Rosen PS, et al. Periodontal regeneration - furcation defects: a consensus report from the AAP Regeneration Workshop. J Periodontol. 2015;86(2 Suppl):S131-3.

24. Cortellini P, Tonetti MS. Microsurgical approach to periodontal regeneration. Initial evaluation in a case cohort. J Periodontol. 2001;72(4):559-69.

25. Harrel SK, Rees TD. Granulation tissue removal in routine and minimally invasive procedures. Compend Contin Educ Dent. 1995;16(9):960, 2, 4 passim.

26. Kao RT, Nares S, Reynolds MA. Periodontal regeneration - intrabony defects: a systematic review from the AAP Regeneration Workshop. J Periodontol. 2015;86(2 Suppl):S77-104.

27. Koop R, Merheb J, Quirynen M. Periodontal regeneration with enamel matrix derivative in reconstructive periodontal therapy: a systematic review. J Periodontol. 2012;83(6):707-20

28. Pretzl B, El Sayed S, Weber D, Eickholz P, Baumer A. Tooth loss in periodontally compromised patients: Results 20 years after active periodontal therapy. J Clin Periodontol. 2018;45(11):1356-64 